\title{
Post-traumatic growth following breast cancer treatment
}

\author{
Ann Marie Corrado, Monica L Molinaro \\ Faculty Reviewer: Marilyn Evans, RN, PhD (Arthur Labatt Family School of Nursing)
}

\begin{abstract}
Worldwide, breast cancer is the most commonly diagnosed cancer amongst women. Currently, in Canada, it is estimated that approximately 1 in 9 women will develop breast cancer at some point in their life, and $\mathbf{1}$ in 30 will die from it. With the growing proportion of women diagnosed with breast cancer each year, there is an expanding body of literature addressing the effect of undergoing breast cancer treatment. The survival rate has increased since the mid-1980s, and as such, more women are in remission from their treatment. To coincide with this, breast cancer literature has begun addressing the effects experienced by women after their treatment. The purpose of this article is to briefly review literature that suggests that women may experience post-traumatic growth after completing breast cancer treatment. Post-traumatic growth, or experiencing positive outcomes as a result of undergoing an extremely negative or traumatic experience, can include developing a new perspective on life, adopting routines to enhance one's quality of life (such as diet and exercise habits), as well as increasing spirituality. This article will outline some potential outcomes of post-traumatic growth, as well as provide insight to health care providers on how to aid in transitioning women with breast cancer from treatment to life in remission.
\end{abstract}

\section{INTRODUCTION}

In 2016, breast cancer accounted for the largest percentage (26\%) of cancers diagnosed in Canadian women. ${ }^{1}$ In particular, approximately 70 women will be diagnosed with breast cancer every day. ${ }^{1}$ Women often experience a multitude of effects during cancer treatment, including physical, psychological, emotional, cognitive, and social effects. ${ }^{2,3}$

As the survival rates for women diagnosed with breast cancer are increasing, ${ }^{1,4}$ research has begun to address the effects of adverse health outcomes experienced by women after treatment has ceased. Many of these health outcomes are psychological in nature, as it is not uncommon to experience mental health effects after experiencing such a traumatic life event. ${ }^{4-6}$ These psychological effects can include: feelings of anger and hostility, depression, anxiety, and post-traumatic stress disorder (PTSD). ${ }^{4,6}$ However, breast cancer survivorship literature has also begun to address outcomes that can be positive for women., ${ }^{4,7}$

Groarke et al. (2016) have acknowledged that over the last twenty years there has been "a growing body of research focused on post-traumatic growth (PTG) in the aftermath of highly stressful life events". Post-traumatic growth, coined originally by Clahoun and Tedeschi (1989), is a term encompassing the potential positive outcomes resulting from an individual's efforts to cope with a traumatic life event. As suggested in previous literature, a traumatic event can also constitute a life-threatening illness, such as cancer. ${ }^{8}$ To Tedeschi and Calhoun (1989) undergoing a traumatic event, otherwise known as a seismic event, allows for the development of coping and adaptation skills when facing such a difficult time. ${ }^{8}$ Therefore, post-traumatic growth occurs as a consequence of developing these skills, and may lead to the creation of more positive outcomes for these individuals. ${ }^{4}$ The purpose of this paper is to provide an understanding of post-traumatic growth in women who experienced breast cancer, and the potential outcomes of post-traumatic growth.

\section{MANIFESTATIONS OF POST-TRAUMATIC GROWTH}

The length of time following trauma after which an individual may start showing signs of post-traumatic growth has been debated in previous literature, as it is questionable as to whether post-traumatic growth, or the depth to which an individual may experience it, is time-dependent. ${ }^{7,9,10}$ While some debated that a specific time period, such as 6-months after treatment, ${ }^{7,9,10}$ is a critical time to assess post-traumatic growth, others state that time after treatment is not an indicator and post-traumatic growth can typically be observed in most cancer survivors at any point past their cancer experiences. ${ }^{11}$ Regardless of the time in which post-traumatic growth could be shown, there are a plethora of positive outcomes associated with post-traumatic growth after breast cancer. These outcomes can include: a better appreciation of life, stronger relationships with family and friends, a deeper sense of spirituality, increased personal strength, and better personal health behaviours., ${ }^{4,7}$

In some cases, women may develop a newfound spirituality, or more pronounced spirituality than before. ${ }^{4,12,13}$ As a result, it is easier for women to make meaning of their breast cancer experiences, as well as develop a new closeness to their spiritual faith., ${ }^{42,13}$ In a study conducted by Fallah et al. (2012), 86.95\% of women had experienced spiritual growth as a result of their breast cancer experiences. $^{4}$

After experiencing a breast cancer diagnosis, many women report developing a new sense of personal strength. Personal strength may hold different meanings to different women. Within this definition, women described having an increased resiliency, self-confidence, or better adaptation and positive coping skills. ${ }^{4,11}$ As well, for some women, personal strength meant developing new goals for them to achieve in their life after cancer treatment.

Another facet of post-traumatic growth is a newfound, or deeper appreciation for life. Many women, after completing treatment, expressed the need to not take life for granted, and to take more time focusing on the aspects of their lives that are most important, rather than worrying about items that were insignificant.,11,12 A newfound appreciation for life is also a multifaceted term, as it 
encompasses factors such as adapting new lifestyles and habits, becoming more grateful for family and friends, and appreciating what is considered truly important to these women., ${ }^{4,12}$ As well, with this new appreciation for life, many women also adapted new routines, such as eating healthier and exercising, in order to extend their lifespan and simultaneously increase its quality. ${ }^{4}$

As previously stated, breast cancer accounts for a large proportion of cancers in Canadian women, however survival rates have increased over the past three decades. ${ }^{1}$ As such, it is imperative for health care providers to understand the multifaceted nature of this disease. While one may believe that treatment and attention to breast cancer ends once there is no physical sign of cancer left in the body, it is necessary to understand that this is not the case. Rather, many women may still experience effects from their experience while in remission, and, as has been shown in many cases, some of these effects are positive in nature. . $^{4,6,711}$

\section{CONCLUSION AND IMPLICATIONS}

Post-traumatic growth has been argued to occur as the result of undergoing a traumatic life experience by developing adaptation skills to handle this event. ${ }^{7}$ Hence, it is important for health care providers to be observant of their patients during the cancer process and be attuned to their levels of stress and distress. ${ }^{7}$ Groarke et al. (2016) found that women who had cancer-specific stress during treatment were at a higher likelihood to develop some semblance of post-traumatic growth when treatment was completed. ${ }^{7}$ However, women who had more "global" stress, or stress unrelated to the cancer, were less likely to develop post-traumatic growth symptoms. ${ }^{7}$ Attention to stress is imperative for health care providers treating breast cancer patients, as they need to pay close attention to their patients and how they cope with their diagnosis and treatment. By doing this, providers can identify women who may need help in transitioning from life during treatment to life post-treatment. However, this is not the case for all women who are diagnosed with breast cancer, as not all women may experience post-traumatic growth. Each woman has a different reaction to their experience and while post-traumatic growth is a potential outcome, it is not the norm, nor should women be expected to acknowledge and express positive attitudes about their breast cancer experience.

Post-traumatic growth is a potential product of undergoing a breast cancer diagnosis and treatment. Within this are multiple outcomes that an individual may experience, including increased personal strength, spirituality, and appreciation for life. While not all women will experience this, it is imperative for health care providers to be attuned to their patients while undergoing breast cancer treatment and beyond, in order to understand how women are affected during and after their breast cancer experiences.

\section{REFERENCES}

1. Canadian Cancer Society [Internet]. Toronto (ON): Canadian Cancer Society c2016. Breast cancer statistics; 2016 [cited 2016 December 10]. Available from http://www.cancer.ca/en/cancer-information/cancer-type/breast/statistics/?region=on .

2. Yarbro CH, Wujcki D, Holmes Gobel B. Cancer nursing: principles and practice. Sudbury, MA: Jones and Bartlett Publishers; 2011. 1940 p.

3. Varricchio C, Pierce M, Hinds, PS, et al. Cancer Source Book for Nurses. Sudbury, MA: Jones and Bartlett Publishers; 2004. 580 p.

4. Fallah R, Keshmir F, Kashani FL, et al. Post-traumatic growth in breast cancer patients: a qualitative phenomenology study. Middle East $\mathrm{J}$ Cancer. 2012;3(2\&3):35-44.

5. Abe O, Abe R, Enomoto K, et al. Effects of chemotherapy and hormonal therapy for early breast cancer on recurrence and 15-year survival: an overview of the randomized trials. Lancet. 2004;365:1687-1717.

6. Ruini C, Vesovelli F. The role of gratitude in breast cancer: its relationship with post-traumatic growth, psychological well-being and distress. J Happiness Stud. 2013;14:263-274.

7. Groarke A, Curtis R, Groarke JM, et al. Post-traumatic growth in breast cancer: how and when do distress and stress contribute? Psycho-Oncol. 2016;1-8.

8. Ruini C, Vescovelli F, Albieri E. Post-traumatic growth in breast cancer survivors: new insights into its relationship with well-being and distress. J Clin Psychol Med S. 2013. 20: 383-391.

9. Kernan WD, Lepore SJ. Searching for and making meaning after breast cancer: prevalence, patterns, and negative affect. Soc Sci Med. 2009;68:1176-82.

10. Tomich PL, Helgeson VS. Is finding something good in the bad always good? Benefit finding among women with breast cancer. Health Psychol. 2004;23:16-23.

11. Lelorain S, Bonnaud-Antignac A, Florin A. Long term posttraumatic growth after breast cancer: prevalence, predictors and relationships with psychological health. J Clin Psychol Med S. 2010;17:14-22.

12. Cordova MJ, Cunningham LL, Carlson CR, et al. Posttraumatic growth following breast cancer: a controlled comparison study. Health Psychol. 2001;20(3):176-85.

13. Mols F, Vingerhoets AJJM, Coebergh JWW, et al. Well-being, posttraumatic growth and benefit finding in long-term breast cancer survivors. Psychol Health. 2009;24(5):583-95. 